\title{
INTERACTION OF PLANETARY NEBULAE WITH THE INTERSTELLAR MEDIUM
}

\author{
K.J. BORKOWSKI \\ Department of Astronomy, University of Maryland, College Park, MD 20742, USA
}

\begin{abstract}
Interaction of planetary nebulae (PNe) with the interstellar medium (ISM) is quite common, in accord with our understanding of the large-scale structure of the ISM. The characteristic signature of the interaction is a dipole asymmetry resulting from a distortion and compression of the nebula in the direction of stellar motion. Studies of interacting PNe provide us with information on the structure of the ISM, and on the evolution of old PNe and PN halos.
\end{abstract}

\section{Introduction}

PNe initially expand freely because their densities exceed the ISM density by orders of magnitude. However, once the PN density drops below a critical value because of the expansion, an interaction takes place between the PN and the ISM.

The strength of the interaction depends on the ISM density and pressure distribution within the layer occupied by PNe. Because most PNe are located outside of the thin H I layer of dense gas in the Galactic plane, the distribution of gas at large distances from the plane is needed. The presence of an extended, $1.5-2 \mathrm{kpc}$ thick layer of ionized gas has been recently established (Reynolds 1991a; Nordgren, Cordes, \& Terzian 1992). Reynolds (1991b) found an average electron density $n_{e}=0.08 \mathrm{~cm}^{-3}$ in this layer. There is also neutral hydrogen at large distances from the Galactic plane (Dickey \& Lockman 1990), with similar (or higher) densities. The third phase of the ISM is composed of the hot $\left(\sim 10^{6} \mathrm{~K}\right)$ ionized gas with a low density of $\sim 0.001 \mathrm{~cm}^{-3}$. Within the Galactic plane, average densities might be several times higher than $n_{0} \sim 0.1 \mathrm{~cm}^{-3}$, typical of the warm neutral and ionized gas above the plane.

Another parameter which strongly influences the interaction is the relative velocity $v_{*}$ between the PN and the ISM, usually dominated by the stellar motion. The ratio of the ISM ram pressure to its thermal pressure is equal to $\left[\left(v_{*}+v_{\text {exp }}\right) / c\right]^{2}$ in the leading (upstream) nebular section, where $v_{\text {exp }}$ denotes the nebular expansion velocity and $c$ is the isothermal sound speed in the ISM. In the warm medium $c=10 \mathrm{~km} \mathrm{~s}^{-1}$, and with the typical values of $v_{*}=60 \mathrm{~km} \mathrm{~s}^{-1}$ and $v_{e x p}=20 \mathrm{~km} \mathrm{~s}^{-1}$, the ISM ram pressure exceeds its thermal pressure by a factor of 60 . The ISM ram pressure is thus usually expected to dominate the interaction process, distorting nebulae in the direction of the stellar motion. 

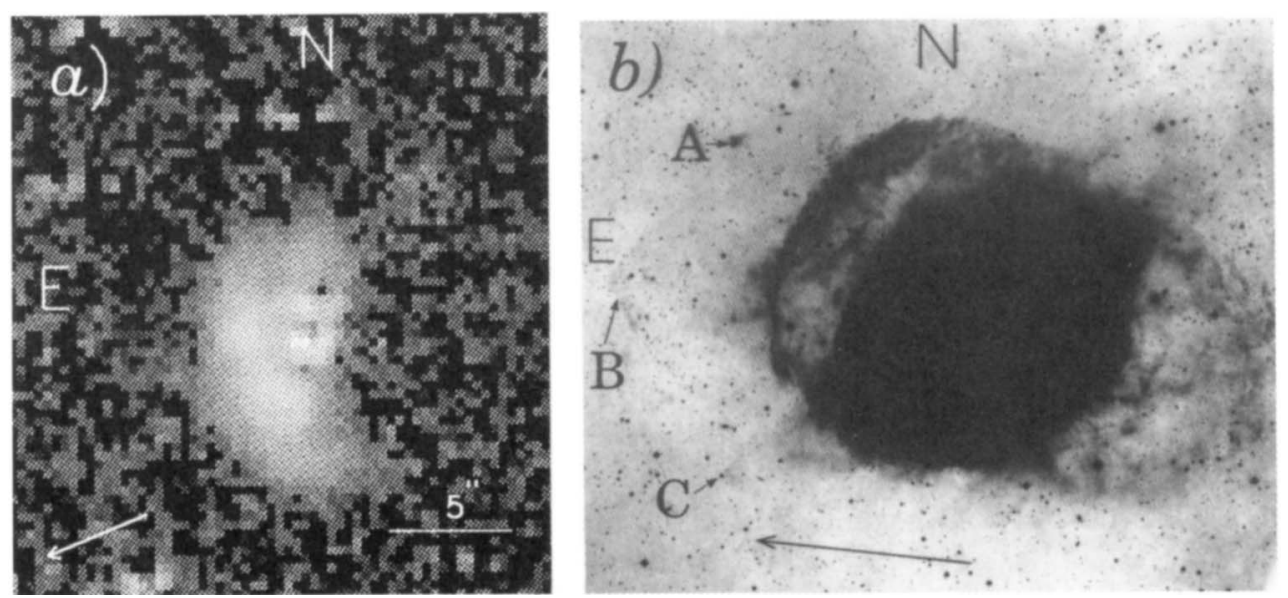

Figure 1: (a) The continuum-subtracted [O III] $\lambda 5007$ image of the M22 PN. Arrow indicates the direction of the transverse cluster motion. A characteristic dipole asymmetry is typical for interacting $\mathrm{PNe}$, with the nebula compressed and distorted in the direction of stellar motion. (b) The Helix nebula in the red light (courtesy of David Malin), showing faint outlying features. Arrow indicates the proper motion direction of the central star.

\section{Observational Evidence}

As suggested by theoretical considerations, a good observational diagnostic for the PN-ISM interaction is a distortion of the nebula in the direction of the stellar motion. This results in a characteristic dipole asymmetry, with the brighter, ram-pressure compressed nebular section located in the leading (upstream) direction. This morphology is clearly seen in interacting PNe PHL932 (Arp \& Scargle 1967), A35 (Jacoby 1981), and S216 (Reynolds 1985). An examination of morphologies of old, nearby $P N e$ revealed a number of nebulae with this dipole asymmetry (Borkowski, Sarazin, \& Soker 1990). The implication is that the PN-ISM interaction should be quite common. This might account for low expansion velocities of old PNe, particularly those located in the Galactic plane (Gieseking, Hippelein, \& Weinberger 1986; Hippelein \& Weinberger 1990). I will now highlight the basic features of the interaction by discussing the most interesting individual cases.

An asymmetric morphology of A35 (Jacoby 1981), with an emission enhancement visible ahead of the fast-moving $\left(125 \mathrm{~km} \mathrm{~s}^{-1}\right)$ star, is characteristic of the PN-ISM interaction. However, a bow-shock structure visible in the [O III] $5007 \AA$ emission line is the most striking nebular feature. Its presence implies that the nebular expansion in the direction of the stellar motion was halted, and the nebular gas is now accelerated past the central star by the ram pressure of the ISM (Jacoby 1981). A35 is in its final evolutionary stage, with the nebula being stripped away from its central star. The nebular r.m.s. electron density is $\sim 10 \mathrm{~cm}^{-3}$ and should be approximately equal to $\left(v_{*} / c\right)^{2} n_{0}=150 n_{0}$. We obtain $n_{0} \sim 0.07 \mathrm{~cm}^{-3}$, in good agreement with expectations. The bow shock forms where the ram pressure of the accelerated $\mathrm{PN}$ gas is balanced by the stellar wind ram pressure. A similar structure is seen in the PN $623+71$, a nebula surrounding a cataclysmic binary (Krautter, 
Klaas, \& Radons 1987; Hollis et al. 1992). Because the central star of A35 is also a close binary, the enhanced stellar winds in close binary systems may play a crucial role in the bow shock formation.

The most spectacular example of a fast-moving, interacting nebula is provided by a hydrogen-poor PN in the globular cluster M22. Figure $1 a$ (Borkowski, Tsvetanov, \& Harrington 1992) demonstrates that because of the high cluster velocity of $200 \mathrm{~km} \mathrm{~s}^{-1}$ (Cudworth 1986), the PN has been very strongly distorted. The estimates of the ambient ISM density are somewhat dependent on modeling, but are again consistent with $n_{0} \sim 0.1 \mathrm{~cm}^{-3}$. The ISM ram pressure will strip the M22 PN from the cluster within several $\times 10^{5} \mathrm{yr}$. Stripping of the M22 PN by the ambient ISM is the first direct evidence for removal of gas from globular clusters.

A different picture of the interaction emerges by looking at the well-known Helix nebula (NGC 7293) in Figure 1b. The motion of the central star (Cudworth, private communication) coincides with the direction determined by the conical filament $\mathrm{B}$ and the cometary object A discovered by Malin (1982). Kwitter, Chu, \& Downes (these proceedings) demonstrated through narrow-band imaging that excitation conditions in these features differ appreciably from those in the nebula proper. These features are most probably sections of the red giant wind (or superwind) which have been compressed in the east by the ram pressure of the ISM. During the course of the PN evolution, a bipolar outflow (Walsh \& Meaburn 1987) expanded in the distorted wind (whose density is higher in the direction of nebular motion), resulting in a strong asymmetry between the two lobes of the outflow. What we are seeing here is an interaction of a PN halo (or an outer shell) with the ISM, which in the Helix nebula has been further modified by the PN dynamics. The halo interaction could have begun long before the $\mathrm{PN}$ formation - for example, a ram-pressure-distorted red giant wind was detected around Mira (Bowers \& Knapp 1988). A systematic investigation of PN halos should reveal more cases like NGC 7293, and some might have been already detected (e. g., NGC 6853 - Borkowski et al. 1990; NGC 6751 - Chu et al. 1991; IC 4593 - Balick et al. 1992; Zucker \& Soker 1992; NGC 3587 - Kwitter et al., these proceedings). Even for spherically symmetric halos, Frank et al. (1990) found that their morphologies require a confining medium with the pressure of $10^{3} \mathrm{~K} \mathrm{~cm}^{-3}$, equal to the thermal pressure of the warm ionized ISM. Therefore, the PN-ISM interaction should influence PN halos.

Among large asymmetric nebulae there are two relatively bright nebulae, A21 (YM29) and S188, located in the Galactic plane. They appear to be moving through dense $\left(n_{0} \geq\right.$ $1 \mathrm{~cm}^{-3}$ ) ISM, which led to an extreme asymmetry, stellar displacements away from the nebular center, and to the creation of a small H II region behind A21 (Weinberger 1989). Recent proper motion measurements by Pier et al. (these proceedings) firmly establish that the extreme asymmetry of A21 is indeed caused by the interaction with the ISM, because the ram-pressure compressed nebular section is located in the direction of the stellar motion. (The same conclusion follows from Pier et al. measurements of the proper motion of the central star of another asymmetric PN A31.) These results demonstrate that proper motion measurements are very useful in studying the interaction of PNe with the ISM.

\section{Summary}

Theoretical work on interacting PNe has been limited. The interaction of a moving PN has been considered in the framework of a thin-shell approximation (Smith 1976; Isaacman 
1979). Soker et al. (1991) performed numerical calculations and refined the analytic theory, finding satisfactory agreement with the thin-shell approximation. The thin-shell approximation breaks down for fast-moving nebulae such as the M22 PN, where the cooling time scale for the shocked ISM is longer than the flow time scale. In this case, Soker et al. (1991) found that the Rayleigh-Taylor instability is likely to fragment the nebular shell into knots, a result which has been confirmed by observations (Fig. 1a).

Available observational data on the interacting nebulae reveal varied and complex dynamical processes which are not explained by the current theory. There is ample evidence for instabilities: linear streaks in A35 and A31, a conical filament and knots with tails in NGC 7293 (Fig. 1b), and strongly filamentary nebulae A21 and S188. More theoretical work is also needed to understand the structure of bow shocks in A35 and the PN $623+71$, the interaction of ionization-bounded nebulae such as S216 (Reynolds 1985), and the time-dependent evolution of miniature H II regions such as seen behind A21.

The current developments have already led to interesting results, which should encourage further studies of interacting PNe. The evolution of old nebulae and of PN halos is commonly affected and may even be governed by the interaction. The interaction of PNe with the ISM is a useful probe of the ISM. With a sufficient effort, it should be possible to determine the filling fraction of the hot ISM phase where the interaction is much weaker than in the warm ISM. For nearby PNe, proper motions of central stars would be very helpful in this respect. Significant improvements are now possible over the last systematic study by Cudworth (1974), as shown by Pier et al. at this conference.

\section{References}

Arp, H., \& Scargle, J. D.: 1967, ApJ 150, 707

Balick, B., Gonzalez, G., \& Frank, A.: 1992, ApJ 392, 582

Borkowski, K. J., Sarazin, C. L., \& Soker, N.: 1990, ApJ 360, 173

Borkowski, K. J., Tsvetanov, Z., \& Harrington, J. P.: 1992, ApJ, in press

Bowers, P. F., \& Knapp, G. R.: 1988, ApJ 332, 299

Chu, Y.-H., Manchado, A., Jacoby, G. H., \& Kwitter, K. B.: 1991, ApJ 376, 150

Cudworth, K. M.: 1974, $A J$ 79, 1384

Cudworth, K. M.: 1986, AJ 92, 348

Dickey, J. M., \& Lockman, F. J.: 1990, ARA\&A 28, 215

Frank, A., Balick, B., \& Riley, J.: 1990, AJ 100, 1903

Gieseking, F., Hippelein, H., \& Weinberger, R.: 1986, $A \& A$ 156, 101

Hippelein, H., \& Weinberger, R.: 1990, $A \& A$ 232, 129

Hollis, J. M., Oliversen, R. J., Wagner, R. M., \& Feibelman, W. A.,: 1992, ApJ 393, 217

Isaacman, R.: 1979, $A \mathscr{E} A$ 77, 327

Jacoby, G. H.: 1981, $A p J$ 244, 903

Krautter, J., Klaas, U., \& Radons, G.: 1987, $A \& A 181,373$

Malin, D. F.: 1982, Sky and Tel. 63, 22

Nordgren, T., Cordes, J., \& Terzian, Y.: 1992, $A J$, in press

Reynolds, R. J.: 1985, ApJ 288, 622

Reynolds, R. J.: 1991a, in IAU Symposium No. 144, The Interstellar Disk/Halo Connection in Galaxies, ed(s)., H. Bloemen, Dordrecht: Kluwer, 67

Reynolds, R. J.: 1991b, ApJ 372, L17

Smith, H.: 1976, MNRAS 175, 419

Soker, N., Borkowski, K. J., \& Sarazin, C. L.: 1991, AJ 102, 1381

Walsh, J. R., \& Meaburn, J.: 1987, MNRAS 224, 885

Weinberger, R.: 1989, in Reviews in Modern Astronomy 2, ed(s)., G. Klare, Springler-Verlag: Berlin; 167

Zucker, D. B., \& Soker, N.: 1992, ApJ, submitted 